\title{
Environmentally-friendly Conductive Cotton Fabric as Flexible Strain Sensor Based on Hot Press Reduced Graphene Oxide
}

Jiesheng Ren ${ }^{\mathrm{a}}$, Chaoxia Wang ${ }^{\mathrm{a}, ~ *}$, Xuan Zhang ${ }^{\mathrm{a}}$, Tian Carey ${ }^{\mathrm{b}}$, Kunlin Chen ${ }^{\mathrm{a}}$, Yunjie Yin $^{\mathrm{a}}$, Felice Torrisi ${ }^{\mathrm{b},}{ }^{* 1}$

${ }^{\text {a }}$ Key Laboratory of Eco-Textile, Ministry of Education, School of Textiles and Clothing, Jiangnan University, Wuxi 214122, China

${ }^{b}$ Cambridge Graphene Centre, University of Cambridge, Cambridge CB3 OFA, U.K.

\begin{abstract}
A flexible conductive cotton fabric was demonstrated by formulation and deposition of a graphene oxide (GO) dispersion onto a cotton fabric by vacuum filtration. The final deposited GO amount was controlled by the concentration and volume of the GO dispersion. The $\mathrm{GO}$ was reduced by a hot press method at $180{ }^{\circ} \mathrm{C}$ for $60 \mathrm{mins}$, and no chemical reductant was needed in both the deposition and reduction processes. The carbon-oxygen ratio increased from 1.77 to 3.72 after the hot press reduction. The asprepared flexible conductive cotton fabric showed a sheet resistance as low as $0.9 \mathrm{k} \Omega / \mathrm{sq}$. The sheet resistance of the conductive cotton fabric only increased from $\sim 0.9 \mathrm{k} \Omega / \mathrm{sq}$ to $\sim 1.2 \mathrm{k} \Omega / \mathrm{sq}$ after 10 washing cycles, exhibiting good washability. The conductive cotton fabric showed viability as a strain sensor even after 400 bending cycles, in which the stable change in the electrical resistance went from $\sim 3500 \mathrm{k} \Omega$ under tensile strain to $\sim 10 \mathrm{k} \Omega$ under compressive strain. This cost-effective and environmentally-friendly method can be easily extended to scalable production of reduced GO based flexible conductive cotton fabrics.
\end{abstract}

\footnotetext{
* Corresponding authors.

E-mail addresses: wangchaoxia@sohu.com (C. Wang), ft242@eng.cam.ac.uk (F. Torrisi).
} 


\section{Introduction}

Conductive textiles have been attracting a broad research attention as they can be used for energy storage [1,2], strain sensors [3] and health care devices [4] enabling wearable textile electronics. Among various textile materials, cotton fabric is one of the most commonly used material for textiles and clothing. Because this material possesses unique flexibility and high porosity [5,6], which makes the cotton fabric comfortable and breathable to wear compared to other synthetic fabrics. More importantly, the chemical structure of cotton fabric possesses numerous active hydroxyl groups, which makes this fabric easy to be modified and also improves the adhesion and distribution of active materials on the fabric $[7,8]$. Conductive cotton fabric has been demonstrated using metal deposition $[9,10]$, or conductive polymer impregnation $[11,12]$. However, the high density, rigid handle, incompatibility of these materials with the human body and the instability of the conductive polymers at room temperature [13] limit their uses.

Graphene has the potential to fulfil most requirements for use in conductive filler, ink and coating [14] thanks to its high carrier mobility [15], mechanical robustness [16], environmental stability and potential for low-cost production. However, due to the lack of functional groups in graphene, the adhesion between graphene and cotton fabric is too weak. Graphene oxide (GO) dispersion in water has been proposed as an alternative conductive coating for cotton fabric due to its abundant oxygen containing groups spreading across the basal planes and the edges [17]. These functional groups make the water-based process realizable. Moreover, the oxygen containing groups both from the GO and cotton fabric form hydrogen bonds with each other which enhances the adhesion. However, the oxidation of graphite disrupts the $\mathrm{sp}^{2}$ structure of graphene planes, making GO flakes intrinsically defective [18] and electrically insulating [19]. Some methods have been reported to fabricate conductive cotton fabrics with reduced GO (r-GO), among which layer by layer assembly as well as dipping-drying method are the most commonly used processes. The GO sheets are absorbed onto the cotton fabric surface or into the strands of the fibers by capillary effect, forming hydrogen bonds with the fabric [20-23]. However, these methods need multiple depositions to deposit substantial GO onto the cotton fabric, making the process not scalable. 
Moreover, the reduction of the GO requires reactive reductants, which are mostly toxic (such as hydriodic acid, titanium trichloride, sodium borohydride and hydrazine) [2427], and may be harmful to the health and environment. Thermal reduction of GO is a greener method and has been demonstrated to be feasible to produce r-GO [28,29]. Ref. [30] reported GO reduction by thermal annealing at temperatures as low as $200{ }^{\circ} \mathrm{C}$ for $2 \mathrm{~h}$, resulting in reduction of the oxygen content to $21 \%$ and increase of the electrical conductivity by 4 orders of magnitude [30]. However thermal reduction of GO to prepare conductive cotton fabrics is avoided to date, because the high reduction temperature and long reduction time tend to critically damage the flexibility and strength of the cotton fabric. Therefore, despite several attempts, developing a sustainable and environmentally-friendly synthesis route of $\mathrm{r}-\mathrm{GO}$ based flexible conductive cotton fabric is still a great challenge.

In this work, we present fabrication of r-GO based conductive flexible cotton fabric and demonstrate its viability as a strain sensor. The conductive cotton fabric was prepared by vacuum filtration of GO water dispersion, followed by a low temperature $\left(180{ }^{\circ} \mathrm{C}\right)$ thermal reduction via hot press method. The morphology of GO and conductive r-GO deposited cotton fabrics, and the reduction process of the GO were characterized (see experimental section). We also investigated the viability of the r-GO deposited cotton fabric as a strain sensor. Furthermore, the washability of the r-GO deposited cotton fabric was evaluated, showing that electrical conductivity was retained for more than 10 washing cycles.

\section{Experimental}

\subsection{Materials}

RS-Aldrich graphite flakes with initial flake size of 100 mesh were purchased from Sigma-Aldrich. Sulfuric acid $\left(\mathrm{H}_{2} \mathrm{SO}_{4}, 95 \sim 98 \%\right)$, phosphoric acid $\left(\mathrm{H}_{3} \mathrm{PO}_{4}, 85 \%\right)$, hydrogen peroxide $\left(\mathrm{H}_{2} \mathrm{O}_{2}, 30 \%\right)$, hydrochloric acid $(\mathrm{HCl}, 36 \sim 38 \%)$ and potassium permanganate $\left(\mathrm{KMnO}_{4}\right)$ were all purchased from Sinopharm Chemical Reagent Co., Ltd. Cotton fabric with yarn count $40 \times 40$ and weighting $130 \mathrm{~g} \mathrm{~m}^{-2}$ was supplied by Wuxi Yihong Textile Co., Ltd. Deionized water was used in the whole experiment.

\section{$2.2 \mathrm{GO}$ preparation}


GO was prepared from graphite flakes according to the improved GO synthesis method [31]. Concentrated $\mathrm{H}_{2} \mathrm{SO}_{4}(72 \mathrm{~mL})$ and $8 \mathrm{~mL} \mathrm{H}_{3} \mathrm{PO}_{4}$ were drop-casted into a flask with $0.6 \mathrm{~g}$ graphite flakes and $3.6 \mathrm{~g} \mathrm{KMnO}_{4}$. The reaction system was stirred at $50{ }^{\circ} \mathrm{C}$ for $12 \mathrm{~h}$ in an oil bath. Then the system was cooled down to room temperature (about $25^{\circ} \mathrm{C}$ ) and poured onto the $200 \mathrm{~g}$ ice which contained $0.6 \mathrm{~mL} 30 \% \mathrm{H}_{2} \mathrm{O}_{2}$ solution. More $\mathrm{H}_{2} \mathrm{O}_{2}$ solution was added into the mixture with mild stirring until the color of the mixture turned golden, which indicated the removal of residual $\mathrm{KMnO}_{4}$ [32]. The mixture was purified by washing in succession with $40 \mathrm{~mL}$ of water and $40 \mathrm{~mL}$ of $30 \%$ $\mathrm{HCl}$, followed by centrifugation. Then the supernatant was decanted away while the remaining was dialyzed with a dialysis bag (molecular weight 8000 10000) in deionized water until the $\mathrm{pH}$ was neutral. The resulting dialyzed dispersion was freeze dried to obtain the GO powder.

\subsection{Deposition of $\mathrm{GO}$ onto the cotton fabric}

The as-prepared GO powder was dispersed in deionized water by bath sonication for 30 mins. The $\mathrm{GO}$ dispersion with a concentration of $5 \mathrm{mg} \mathrm{mL}^{-1}$ was then deposited onto the cotton fabric using a vacuum filtration method. In order to slow down the filtration speed and prevent the loss of the GO sheets, a microporous membrane with pore size $0.45 \mu \mathrm{m}$ was placed underneath the cotton fabric during the filtration process.

\subsection{GO thermal reduction}

The cotton fabric deposited with GO was hot pressed with a PTJ38 flat heat press machine (Yiwu Maike Digital Imaging Co., Ltd, China), in which the GO was reduced to $\mathrm{r}-\mathrm{GO}$ under thermal condition. Considering that the high reduction temperature may lead to cotton fabric degradation, the reduction temperature was set at $180{ }^{\circ} \mathrm{C}$ for the experiment. The reduction time varied from 30 mins to 180 mins.

\subsection{Characterization and Measurement}

The scanning electron microscope (SEM) images of the cotton fabric before and after the GO deposition and GO reduction were acquired on a SU1510 SEM unit (Hitachi, Ltd, Japan). The field emission scanning electron microscope (FE-SEM) and scanning probe microscope (SPM) images of the GO sample were performed on a sigma HD FESEM unit (Carl Zeiss Microscopy, Germany) and SPM9700 unit (SHIMADZU 
corporation, Japan), respectively. The Raman spectra were acquired using a Renishaw inVia Raman spectrometer (Renishaw PLC, UK) with a $514 \mathrm{~nm}$ laser. The X-ray photoelectron spectroscopy (XPS) was performed using a PHI 5000C ECSA (PerkinElmer corporation, USA). The K/S value which used to represent the amount of dye fixation or dye content of a given textiles was measured to display the relative color change [33] of the conductive fabric with a Xrite-8400 spectrophotometer (X-Rite Co., Ltd, USA).

The washing test of the r-GO deposited cotton fabric was carried out on a Labortex oscillating type dyeing machine (Rapid Co., Ltd, China). For each washing cycle, the sample was washed with $100 \mathrm{~mL}$ deionized water containing $2 \mathrm{mg} \mathrm{mL}^{-1}$ sodium carbonate and $5 \mathrm{mg} \mathrm{mL}^{-1}$ soap at $60{ }^{\circ} \mathrm{C}$ for 30 mins. The sheet resistance of the r-GO deposited cotton fabric was measured using a SZT-2A four-point probe tester (Suzhou Tongchuang Electronics Co., Ltd, China). The fabric resistance during the bending process was recorded with a Keithley 2400 source/meter (Tektronix Inc., USA).

\section{Results and Discussion}

\subsection{GO preparation}

Fig. 1(a) shows GO flakes deposited on an Xindi tinfoil substrate (Wuxi Xindi Plastic and Paper Product Co., Ltd, China). The lateral size of the flakes ranges from tens of nanometers to hundreds of nanometers. In Fig. 1(b), the GO sheet presents a thickness ranging from $0.9 \mathrm{~nm}$ to $1.5 \mathrm{~nm}$ under the effect of the rough substrate (substrate roughness, $\mathrm{Rq}=0.2 \mathrm{~nm}$ ) and wrinkles formed by the sheet itself. The average thickness of the sheets is measured to be $1.2 \mathrm{~nm}$. Typically, due to the existence of epoxy, carboxyl and hydroxyl groups on both sides of GO sheets, the thickness of monolayer GO varies between $0.8 \mathrm{~nm}$ and $1.2 \mathrm{~nm}[34,35]$. The interlayer distance of GO ranges from $0.6 \mathrm{~nm}$ to $1.2 \mathrm{~nm}[36]$. So the height of the sheet corresponds to exactly one-layer GO. 

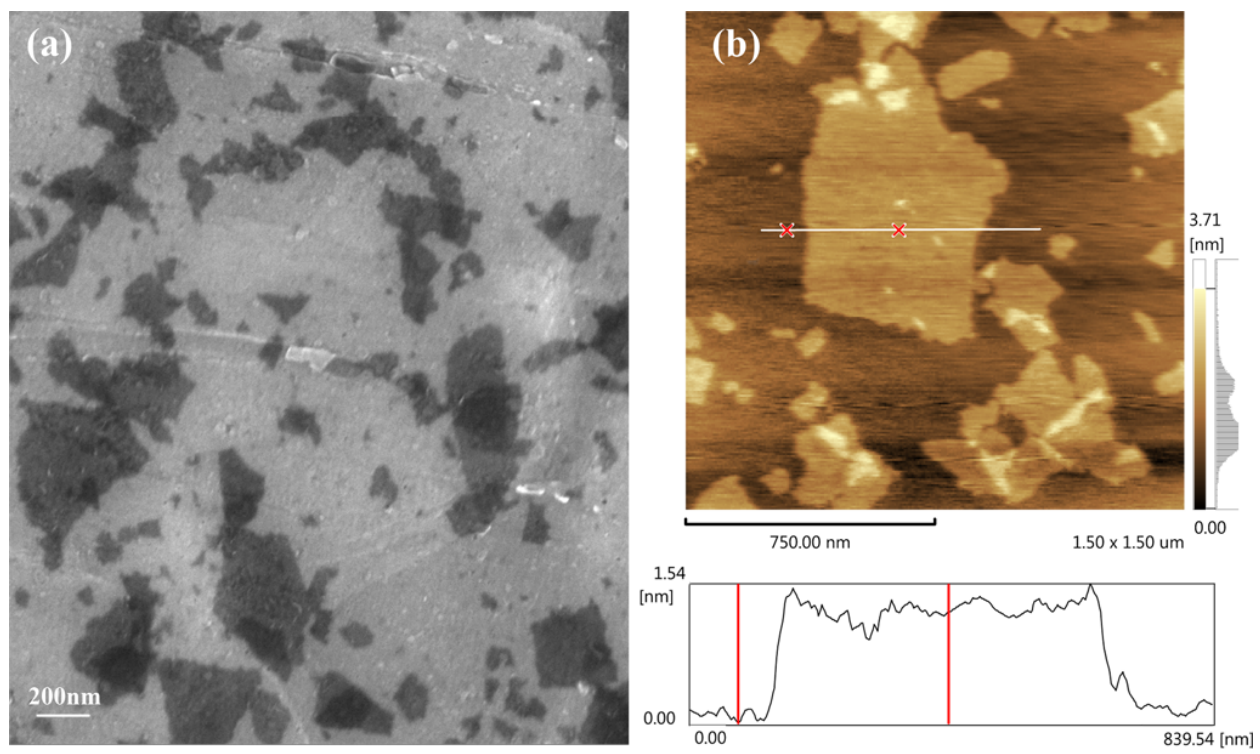

Fig. 1. (a) FE-SEM image of GO sheets, (b) a typical SPM image of GO sheet, showing the average thickness of the GO sheet is $1.2 \mathrm{~nm}$.

The Raman spectrum is strongly sensitive to the electronic structure of carbon materials and has shown to be an essential tool to characterize graphene [37]. Raman spectra of the raw material graphite and the as-prepared GO are shown in Fig. 2. The graphite spectrum (Fig.2(a)) shows two typical major peaks: the G peak at $\sim 1581 \mathrm{~cm}^{-1}$ and the 2D peak at $\sim 2740 \mathrm{~cm}^{-1}$. A weak D peak appears at approximately $1358 \mathrm{~cm}^{-1}$, caused by the edges of the graphite (100 mesh flake size). The $\mathrm{G}$ peak corresponds to the high frequency $\mathrm{E}_{2 \mathrm{~g}}$ phonon at $\Gamma$, and the $\mathrm{D}$ peak is due to the breathing modes of six-atom rings and requires a defect for its activation $[38,39]$. The $2 \mathrm{D}$ peak is the $\mathrm{D}$ peak overtone. Since $2 \mathrm{D}$ originates from a process where momentum conservation is satisfied by two phonons with opposite wave vectors, no defects are required for their activation, and are thus always present [37]. Besides the fluorescence background between $2000 \mathrm{~cm}^{-1}$ and $3200 \mathrm{~cm}^{-1}$, the GO spectrum (Fig. 2(b)) shows a broad D peak at $1355 \mathrm{~cm}^{-1}$ and $\mathrm{G}$ peak at $1607 \mathrm{~cm}^{-1}$. In this case, the $\mathrm{G}$ peak shows a blue shift of 26 $\mathrm{cm}^{-1}$ with respect to the spectrum of graphite, this can be attributed to the presence of the isolated double bonds resulting from their higher resonant frequencies. The full width half maximum $\mathrm{G}$ peak for the GO flakes corresponds to $73 \mathrm{~cm}^{-1}$, which is $\sim 4.5$ times higher than that of graphite, due to disordered distribution of scattering point defects [40]. The oxidation is also confirmed by the ratio of the D peak and G peak 
intensity $\mathrm{I}(\mathrm{D}) / \mathrm{I}(\mathrm{G})$ and full width half maximum $\mathrm{D}$ peak, being $\sim 0.92$ and $\sim 107 \mathrm{~cm}^{-1}$ respectively, which raise from the defects both in the basal planes and at the edges of the GO flakes $[41,42]$.

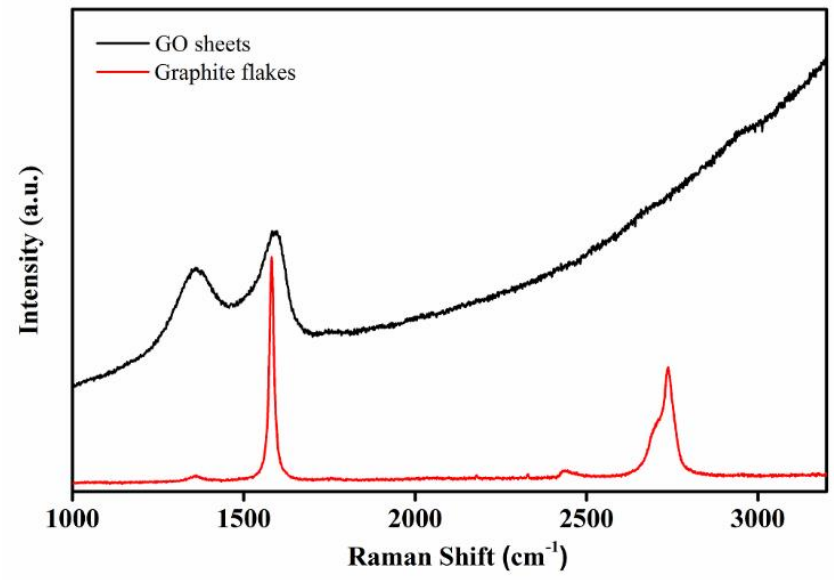

Fig. 2. Raman spectra of the raw material graphite flakes and the as-prepared GO sheets deposited on glass slide.

\subsection{The r-GO deposited cotton fabric}

The GO sheets were deposited onto the cotton fabric through vacuum filtration process of the GO dispersion, and then reduced with a heat press machine as shown in Fig. 3(a). The control cotton fabric shows a white color as shown in Fig. 3(b). After the $\mathrm{GO}$ deposition, the cotton fabric is dried in the oven at $60{ }^{\circ} \mathrm{C}$ for $2 \mathrm{~h}$ to remove the residual water, resulting in a dark brown uniform deposited layer as shown in Fig. 3(c). Subsequently, after the hot press process the color of the deposited layer changes from dark brown to black and shows a quite smoother surface (Fig. 3(d)) with respect to the GO deposited cotton fabric, indicating the partial GO reduction. The SEM micrograph of the control cotton fabric (Fig. 3(e)) shows a typically fibrous structure with the fibers twisting with each other, producing a quite rough fabric surface. During the deposition process, the GO sheets are expected to be deposited onto the cotton fabric surface and trapped between the gaps of the fibers. Fig. 3(f) shows how the fabric surface is uniformly covered with the GO deposited layer and both the fibrous structure and the gaps between the fibers can hardly be seen after the deposition. During the hot press treatment, the GO deposited layer is gradually reduced on the cotton fabric surface and conforms to the morphology of the fabric (Fig. 3(g)). This may be attributed to a 
shrinkage of the r-GO deposited layer as the water is evaporated and the GO is reduced [43]. In contrast to Fig. 3(f), the fabric structure after GO reduction is more clearly visible indicating the r-GO sheets compactly wrap onto the fibers as evident in Fig. 3(g). The cotton fiber microstructure is clearly visible in Fig. 3(h) by SEM at a higher magnification, while it appears covered by GO and r-GO in Figs. 3(i, j), with a more evident surface topography in the r-GO case in Fig. 3(j) as opposed to GO in Fig. 3(i). The SEM images showing the changes in the cross section of cotton fabric after the deposition and the hot press treatment are presented in Fig. S3.
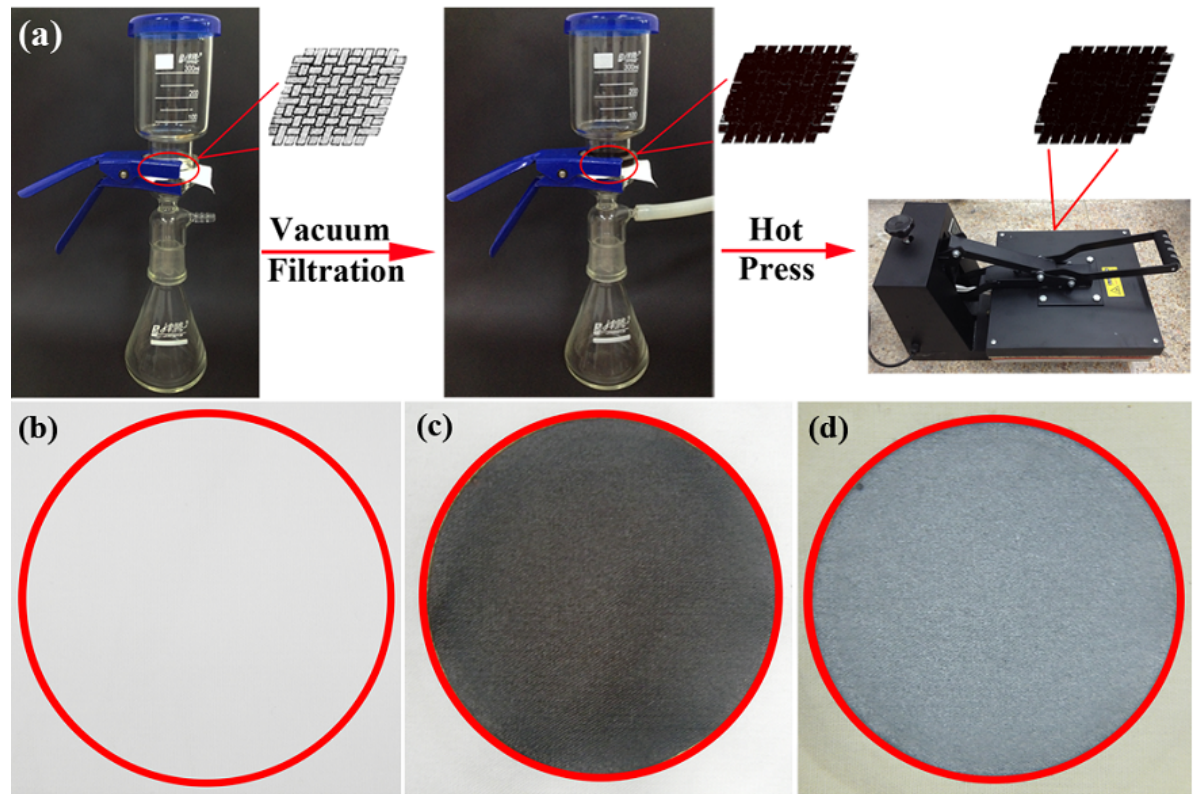

(c)
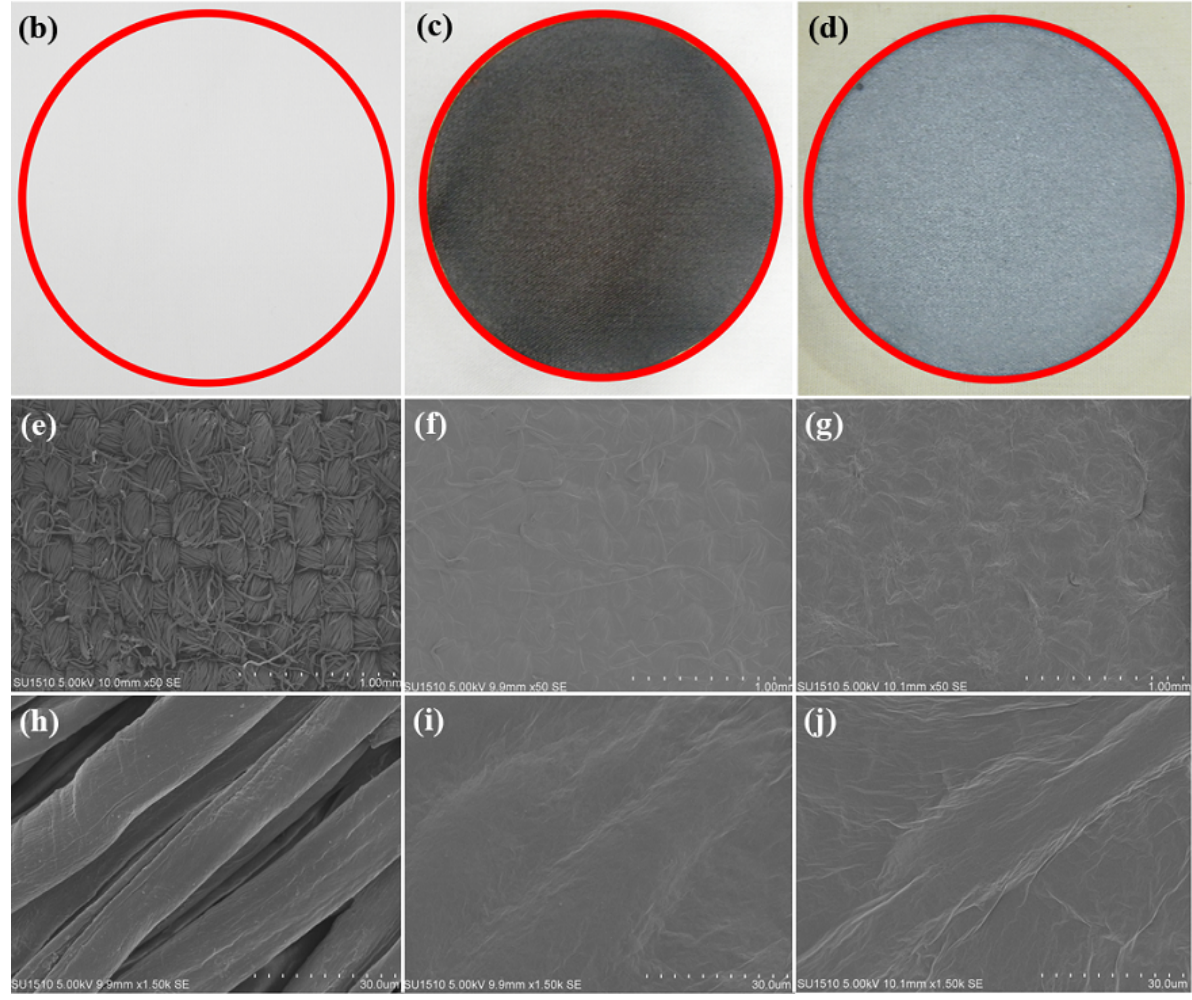

Fig. 3. Photographs of (a) the fabrication process of the r-GO deposited cotton fabric with vacuum filtration and hot press treatment, and (b) control cotton fabric, (c) GO deposited cotton fabric, (d) r-GO deposited cotton fabric hot pressed at $180^{\circ} \mathrm{C}$ for 60 mins, SEM images of (e, h) control fabric, (f, i) GO deposited cotton fabric, (g, j) r-GO deposited cotton fabric. 
The as-prepared r-GO deposited cotton fabric exhibits a good flexibility and bendability (bending example shown in Fig. 4(a)). The sheet resistance of the r-GO deposited fabric measured with a standard four-point probe method is $\sim 0.9 \mathrm{k} \Omega / \mathrm{sq}$, while the sheet resistance of GO deposited fabric is higher than $1000 \mathrm{k} \Omega / \mathrm{sq}$ (which is the high upper limit of the instrument). The conductive r-GO deposited cotton fabric can be implemented as a conductive interconnection. In Fig. 4(b) we show the conductive cotton fabric which closes the circuit of two $1.5 \mathrm{~V}$ batteries and three parallel connected light-emitting diodes (LEDs), turning on the LEDs.

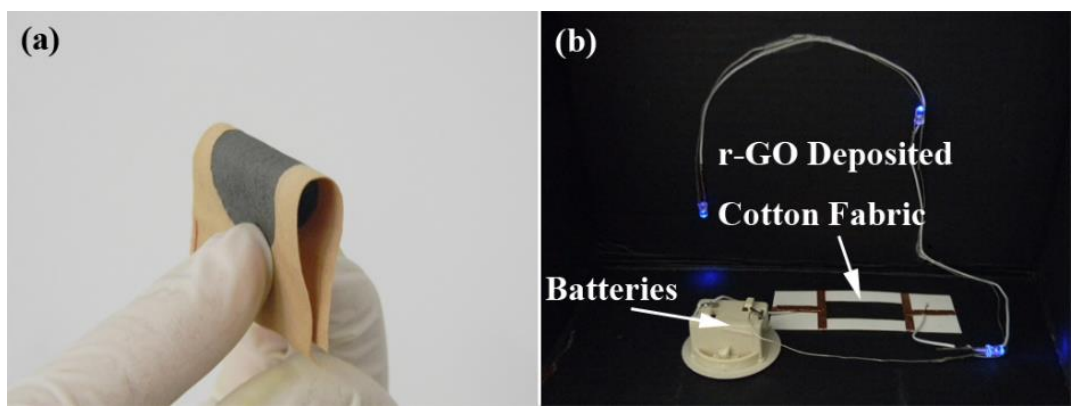

Fig. 4. Pictures of the conductive r-GO deposited cotton fabric hot pressed at $180^{\circ} \mathrm{C}$ for 60 mins (a) can be bended easily and, (b) lightens light-emitting diodes (LEDs) with two $1.5 \mathrm{~V}$ batteries in a circuit.

The amount of the deposited GO on the fabric can be controllable with the vacuum filtration method. The deposited GO amount as function of the vacuum filtered GO volume is shown in Fig. 5, which shows almost a linear relation with the GO amount going from $17 \mathrm{mg}$ for $5 \mathrm{~mL}$ to $56 \mathrm{mg}$ for $20 \mathrm{~mL}$. Besides, the color of the deposited fabrics becomes darker with the deposited GO volume increasing from $5 \mathrm{~mL}$ to $20 \mathrm{~mL}$, as shown in the insert in Fig. 5. During the hot press process, the high temperature can facilitate the thermal deoxygenation of GO. Thus, the oxygen containing groups are gradually removed and the $\mathrm{sp}^{2}$ structure of graphene are partially restored [44]. In order to verify the effect of the hot press treatment on the electrical properties, the sheet resistance was measured on all samples. As shown in Fig. 6, the sheet resistance of these r-GO deposited fabrics decreases dramatically by almost two orders of magnitude within the first 60 mins, where the samples with 15 and $20 \mathrm{~mL}$ GO loading reach lower than $5 \mathrm{k} \Omega /$ sq. However, the sheet resistance of the fabrics with 10,15 and $20 \mathrm{~mL} \mathrm{GO}$ 
loading only shows a small change from $5 \mathrm{k} \Omega / \mathrm{sq}$ to $1 \mathrm{k} \Omega / \mathrm{sq}$ due to the small reduction degree of the GO with longer hot press time, while the sheet resistance of the fabric with $5 \mathrm{~mL}$ GO loading is higher and decreases from $21 \mathrm{k} \Omega / \mathrm{sq}$ to $14 \mathrm{k} \Omega / \mathrm{sq}$. The cotton fabric deposited with $15 \mathrm{~mL} \mathrm{GO}$ and hot pressed for 60 mins was used in the following experiments given the trade-off between electrical conductivity and short preparation time.

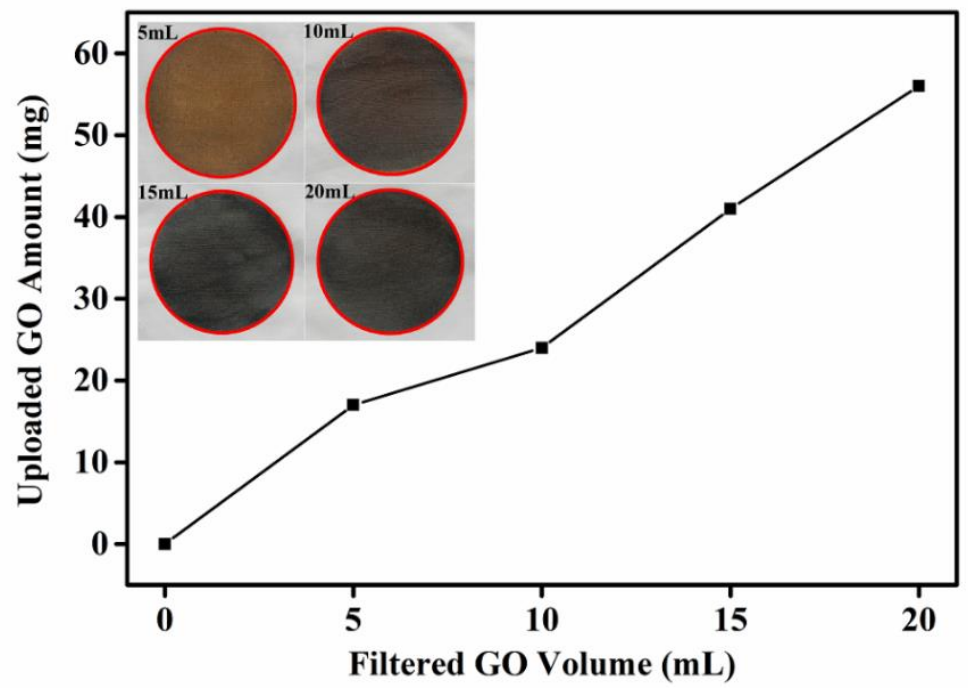

Fig. 5. The uploaded GO amount on the cotton fabric exhibits almost a linear relation with the filtered GO volume and the insert shows the color of the cotton fabrics with different amount of deposited GO (5 mL, $10 \mathrm{~mL}, 15 \mathrm{~mL}$ and $20 \mathrm{~mL})$.

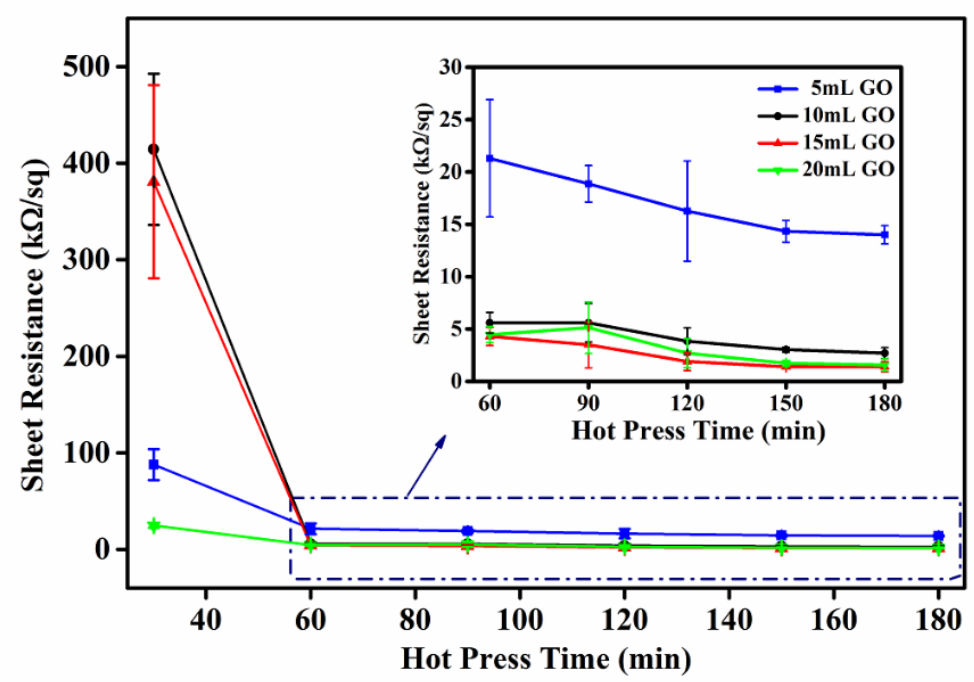

Fig. 6. The sheet resistance of r-GO deposited cotton fabrics with different hot press time and the insert shows a zoom-in of the part in the dashed line frame. 
The reduction of the deposited GO layer at different reduction time upon the hot press treatment is characterized by the Raman spectra (in the region between 1000 to $1800 \mathrm{~cm}^{-1}$ ) as shown in Fig. 7. The spectrum of the control cotton fabric shows a featureless fluorescence [21]. In contrast, the Raman spectra of the GO deposited cotton fabric samples both before and after the hot press process show a D peak at $1360 \mathrm{~cm}^{-1}$ and a $\mathrm{G}$ peak located at $\sim 1600 \mathrm{~cm}^{-1}$. The intensity ratio of $\mathrm{I}(\mathrm{D}) / \mathrm{I}(\mathrm{G})$ (inset in Fig.7) ranges between 0.92 and 0.94 after 5 min hot press treatment followed by a decrease to $\sim 0.89$ from 60 min to $180 \mathrm{~min}$ of hot press treatment, suggesting a slight increase in the average size of the $\mathrm{sp}^{2}$ domain and the realization of deoxygenation in the r-GO deposited layer [35].We note that both the values of the D peak intensity and the $\mathrm{I}(\mathrm{D}) / \mathrm{I}(\mathrm{G})$ for the same samples hot pressed for longer time than 60 mins show no relevant difference with the ones at 60 mins, which is consistent with the small sheet resistance change of the r-GO deposited cotton fabric at longer hot press time than 60 mins with respect to the larger initial sheet resistance change in Fig. 6. These results suggest that the GO is partially reduced by the hot press method in the initial 60 mins and a longer hot press time only shows little effect on the reduction degree. We also used K/S value of the sample to characterize the reduction of GO. As shown in Fig. S2 in the supplementary information, the K/S value of the r-GO deposited cotton fabric tends to be stable after 60 mins hot press, corroborating the small partial GO reduction degree taking place for hot press times longer than 60 mins.

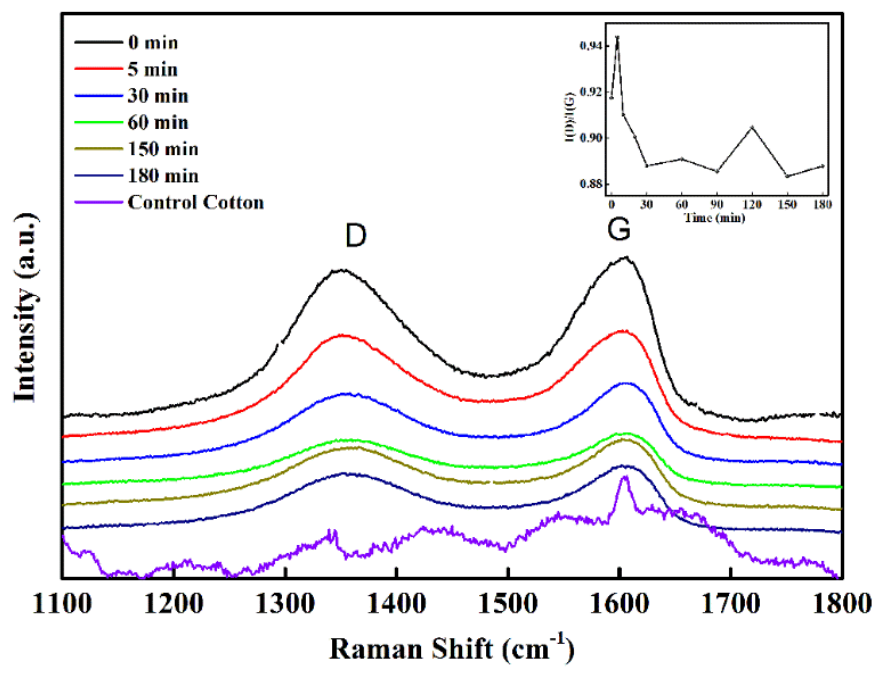


Fig. 7. Raman spectra of the control cotton fabric and the GO deposited fabric hot pressed for different time. Inset: $\mathrm{I}(\mathrm{D}) / \mathrm{I}(\mathrm{G})$ as a function of the hot pressed time

The partial reduction of GO was further evidenced by the XPS spectra. The C1s spectrum of the GO deposited layer in Fig. 8 (a) reveals that the as-prepared GO consists of four components arising from $\mathrm{C}=\mathrm{C} / \mathrm{C}-\mathrm{C}(284.8 \mathrm{eV})$ groups, $\mathrm{C}-\mathrm{O}(286.9 \mathrm{eV})$ groups, $\mathrm{C}=\mathrm{O}(288 \mathrm{eV})$ groups and $\mathrm{O}-\mathrm{C}=\mathrm{O}(289.2 \mathrm{eV})$ groups $[45,46]$. In contrast to the $\mathrm{GO}$ spectrum, the intensity of the $\mathrm{C}=\mathrm{O}$ groups and $\mathrm{O}-\mathrm{C}=\mathrm{O}$ groups are much lower for $\mathrm{r}$ GO as shown in Fig. 8(b), while the intensity of $\mathrm{C}-\mathrm{O}$ groups only shows a slight decrease. This is consistent with previous reports about GO reduction at around $180{ }^{\circ} \mathrm{C}$ [19]. A previous study has already proven that the hydroxyl groups and epoxy groups located at the interior of the GO sheets are not stable and subject to dissociation or migration to the edges of the aromatic domains [47], while the carboxyl groups and the carbonyl groups are reduced slowly at temperature ranging within 170 to $200{ }^{\circ} \mathrm{C}$ [48], which is consistent with our result. In Fig. S4, the XPS spectra of the GO deposited cotton fabrics acquired over a wider range of binding energy also show that after the hot press process at $180^{\circ} \mathrm{C}$ for 60 mins, the intensity of $\mathrm{C} 1 \mathrm{~s}$ increases and the intensity of $\mathrm{O}$ 1s decreases. The element summary indicates the oxygen content of the deposited layer decreases from $35.75 \%$ to $20.03 \%$, while the carbon content increases from $63.26 \%$ to $74.59 \%$. And the carbon-oxygen ratio increases from 1.77 to 3.72. Both the decrease of oxygen containing groups and the changes of the element ratios of the XPS characterization prove the hot press method is an effective method for GO reduction. Additional, the EDX characterization results (see Fig. S5) indicate that the reduction happens not only on the surface but also in the deeper part of the deposited layer.
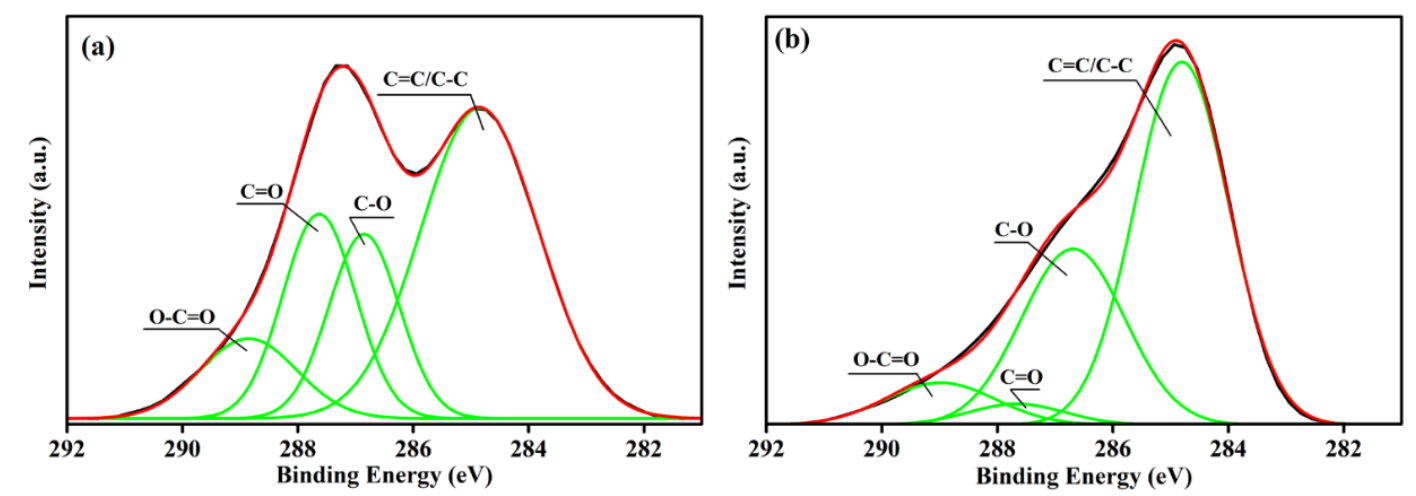
Fig. $8 \mathrm{C}$ 1s of XPS spectra of the (a) GO and (b) r-GO deposited layers

\subsection{Flexible strain sensor}

The r-GO deposited cotton fabric can be used as a strain sensor. The r-GO deposited cotton fabric with $3 \mathrm{~cm}$ long and $1 \mathrm{~cm}$ wide was fixed on a paper substrate with two ends connected to copper tapes as contact electrodes. The conductive r-GO deposited cotton fabric is first subjected to tensile strain generated from the strain applied upon bending with concave down conditions as shown in Fig. 9(a) inserts. As a result, the resistance of the $\mathrm{r}-\mathrm{GO}$ deposited cotton fabric under different bending radii (from $5 \mathrm{~cm}$ to $2.5 \mathrm{~cm}$ ) increases from $16.7 \mathrm{k} \Omega$ to $167.9 \mathrm{k} \Omega$. On the contrary, when the $\mathrm{r}-\mathrm{GO}$ deposited cotton fabric is subjected to compressive strain from the strain applied upon bending with concave up conditions as shown in Fig. 9(c) inserts, the resistance decreases from $22.6 \mathrm{k} \Omega$ to $9.8 \mathrm{k} \Omega$ corresponding to bending radii from $5 \mathrm{~cm}$ to $2.5 \mathrm{~cm}$.

To gain further insight into the mechanism resulting in the resistance change, we performed SEM investigation of the r-GO deposited cotton fabric under tensile (Fig. 9(b)) and compressive (Fig. 9(d)) strain. Fig. 9(b) shows fractures of the r-GO deposited layer under tensile strain, revealing the woven fiber structure underneath. Such fractures are attributed to the fracture strains of the r-GO deposited layer and the cotton fabric. Indeed, the higher flexibility of the cotton fabric given by the fibers form factor allows change in shape and the distance between fibers under tension or compression condition. Conversely, the r-GO deposited layer is more rigid than the cotton fabric, and it can be only accommodated by cracking and subsequent sliding, separating and rearranging of the fractured islands of r-GO films which can cause the change of the resistance. A similar behavior has been reported for graphene thin films on stretchable Poly-dimethyl siloxane substrate [49].

In general, in tensile strain conditions, the distance between the woven fibers tends to increase as sketched in Fig. 10. Subsequently the r-GO layer is subjected to a positive strain which results in cracks of the r-GO deposited layer as shown in Fig. 9(b). The resistance changes of the r-GO deposited cotton fabric are ultimately based on the cracks of r-GO coating layer which in this case results in the resistance increase. On the contrary, in compressive strain conditions the distance between the cotton fibers 
becomes smaller as shown in Fig. 10, leading to cracks narrowing and the layers of $\mathrm{r}^{-}$ GO film to overlap (see Fig. 9(d)), thus decreasing the resistance.
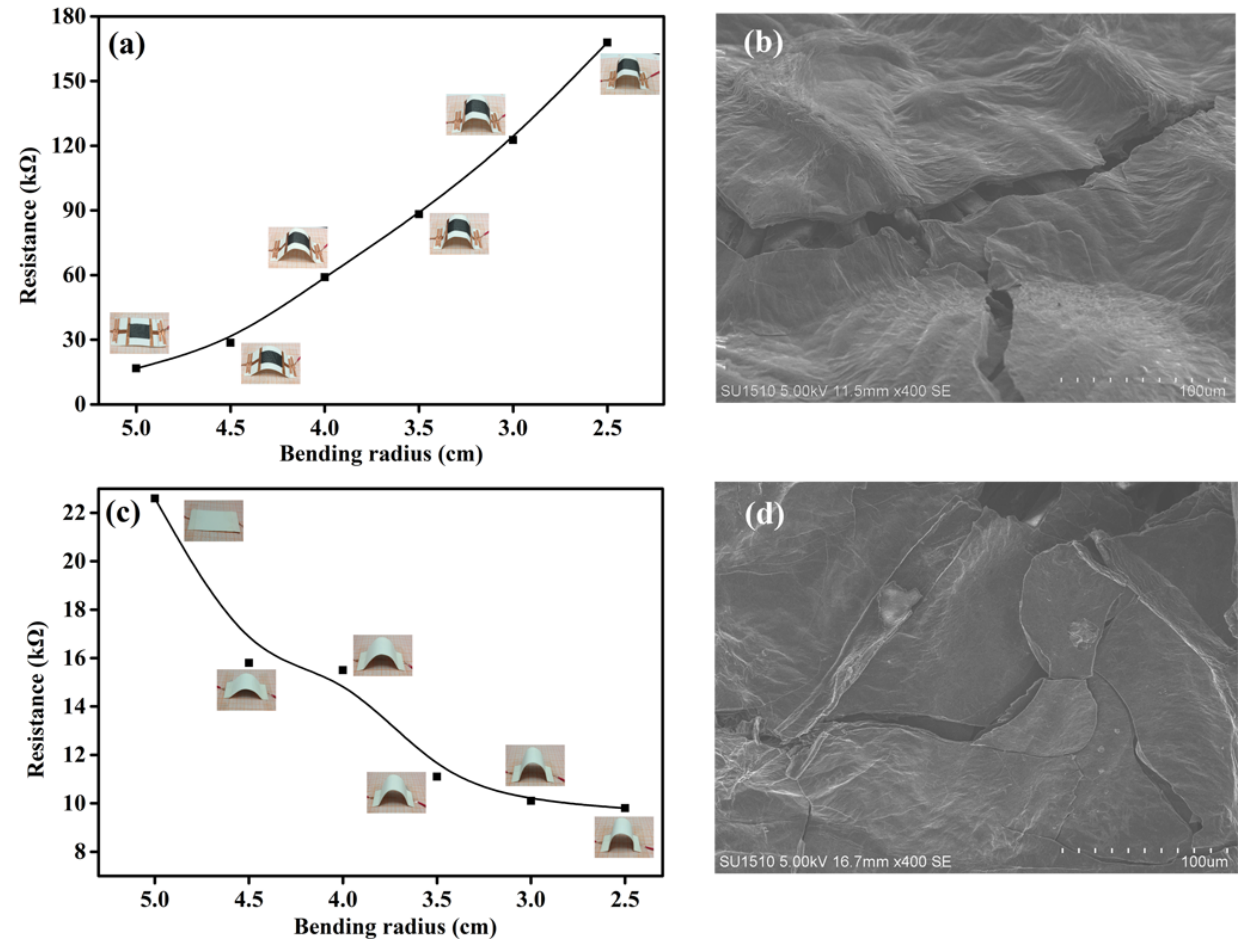

Fig. 9. The resistance of the r-GO deposited fabrics under tensile and compressive strain with the corresponding SEM images (a) the resistance of the r-GO deposited cotton fabric under tensile strain, (b) SEM image shows cracks on the r-GO deposited layer under tensile strain, (c) the resistance of the r-GO deposited cotton fabric under compressive strain, (d) SEM image shows overlaps on the r-GO deposited layer under compressive strain.
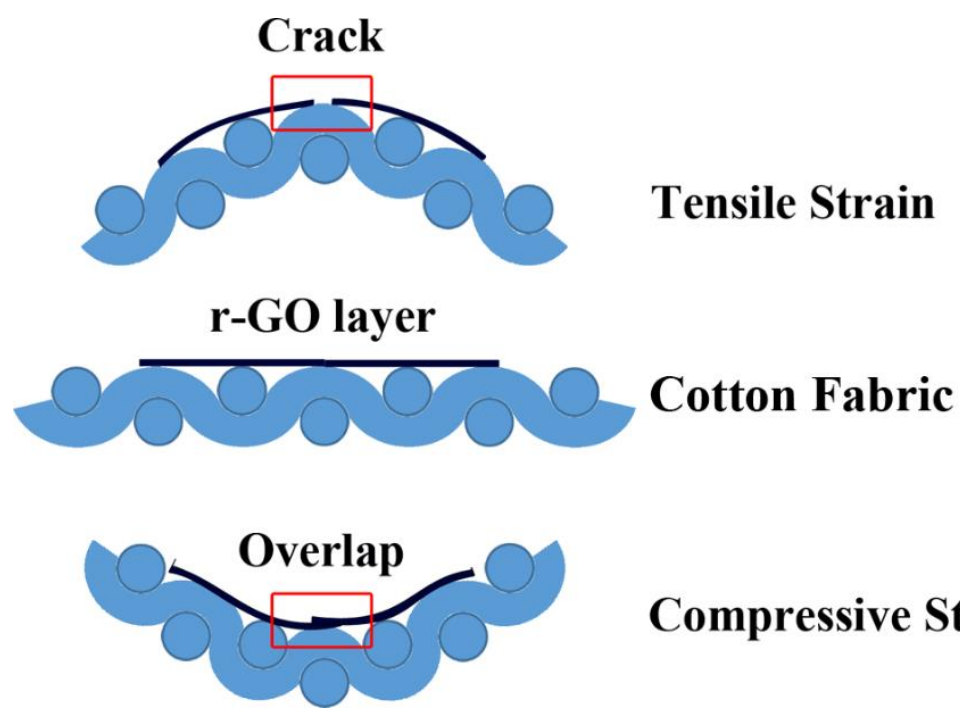

\section{Compressive Strain}

Fig. 10. The r-GO deposited layer on the cotton fabrics cracks and overlaps under tensile and compressive strain. 
Washability is an essential property of conductive textiles [50, 51]. During the washing process, the vessel containing the r-GO deposited cotton fabric and the washing bath was shaken by the dyeing machine, and the oscillation of the washing bath simulated the stress generated during the standard washing cycles, which may affect the integrity and continuity of the deposited layer, resulting in the increase of the sheet resistance [52]. The sheet resistance of the r-GO deposited cotton fabric after washing test was measured to evaluate any effect of the washing cycles on the electrical conductivity. As shown in Fig. 11, the sheet resistance rises slightly with the number of washing cycles, going from $0.9 \mathrm{k} \Omega / \mathrm{sq}$ to $1.2 \mathrm{k} \Omega / \mathrm{sq}$. And after 10 washing cycles, it is still lower than $2 \mathrm{k} \Omega / \mathrm{sq}$, indicating the washing cycles do not obviously affect the conductivity of the r-GO deposited cotton fabric.

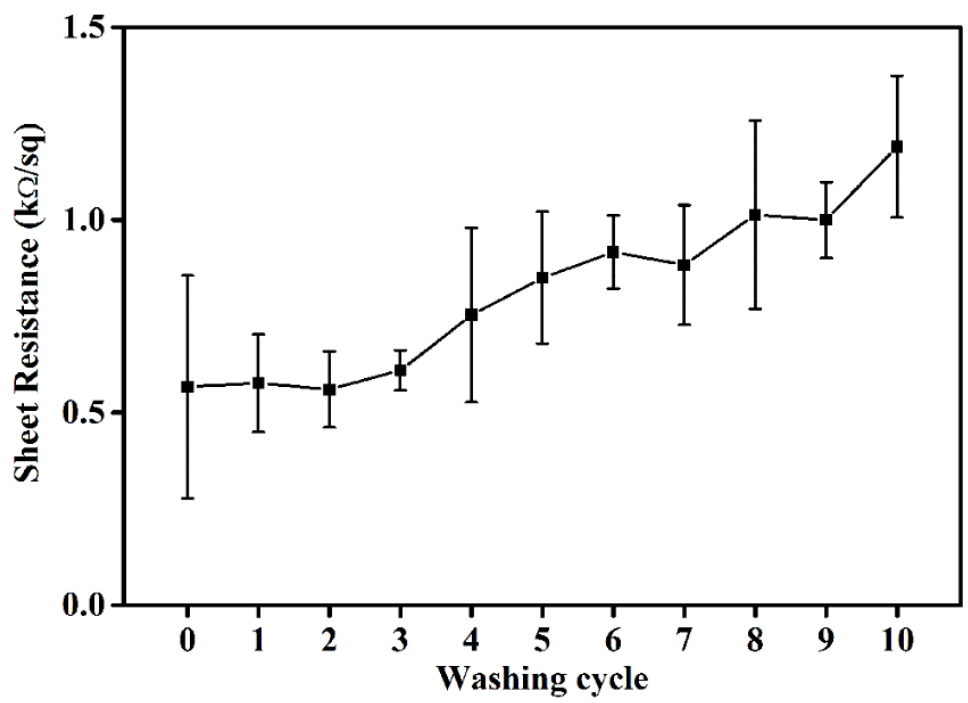

Fig. 11. The sheet resistance change of r-GO deposited cotton fabric as a function of the number of washing cycles.

The r-GO deposited cotton fabric is then laid alongside the wrist (Fig. 12 (a) top insert) to investigate the viability as a strain sensor. The r-GO deposited cotton fabric was pasted onto the wrist with the both ends connected to a Keithley source/meter. Then the resistance change of the r-GO deposited cotton fabric during the movement was recorded. It is found that the resistance of the r-GO deposited fabric rises up to 3500 $\mathrm{k} \Omega$ when wrist bends downwards (Fig. $12(\mathrm{a}, \mathrm{b})$ ), we expect this to happen because the r-GO deposited layer cracks under tensile strain as shown in Fig. 9. On the other side, the resistance decreases to about $10 \mathrm{k} \Omega$ (Fig. 12 (a, b)) when wrist bends upwards 
caused by the overlap of the deposited r-GO cracked layers (see Fig. 9). More importantly, the resistance change follows repeatedly the wrist bending upwards and downwards even after about 400 bending cycles. The SEM images in Fig. S6 show the cracks and the overlaps of the deposited layer after about 400 bending cycles, in which the degree of the cracks and overlaps increases with increasing of the bending cycles. All this indicates the viability of the r-GO deposited cotton fabric as a strain sensor.
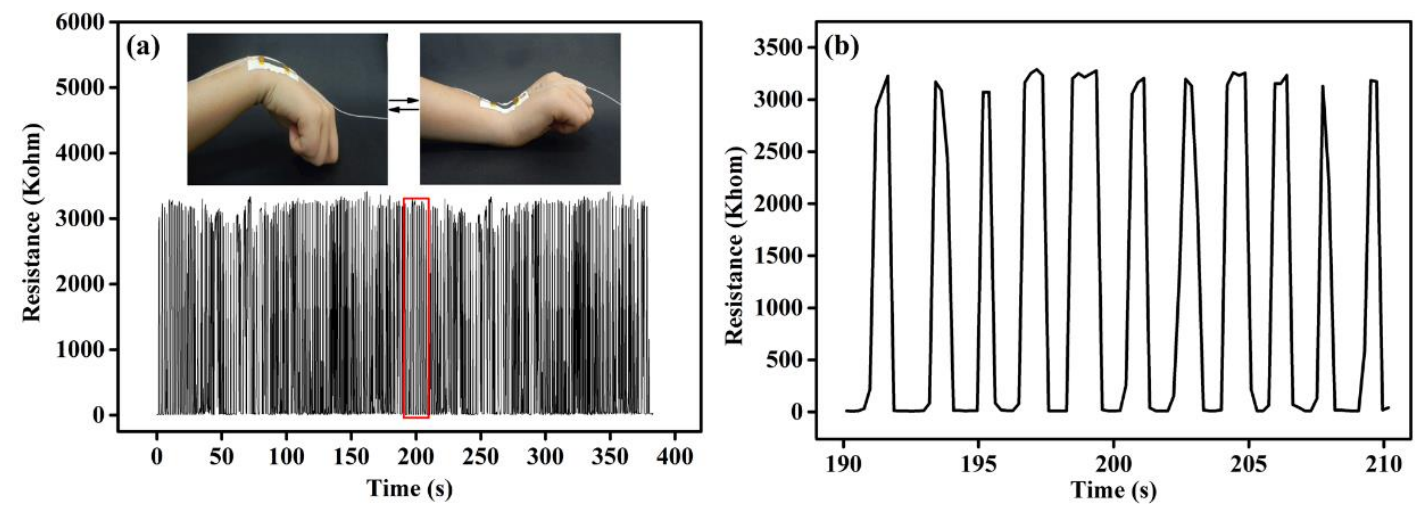

Fig. 12. (a) The resistance of the strain sensor shows regular change in monitoring the wrist bending downwards and upwards, (b) an enlarged part from $190 \mathrm{~s}$ to $210 \mathrm{~s}$.

\section{Conclusions}

In summary, the flexible conductive r-GO deposited cotton fabric that can be used as strain sensor was fabricated via the vacuum filtration of GO solution (which are now readily available on the market) and hot press reduction method. The vacuum filtration process allowed to control the deposited GO amount by changing the GO dispersion volume. The hot press method partially reduced the GO to r-GO with a hot press reduction process at $180{ }^{\circ} \mathrm{C}$ for 60 mins, thus proposing a green protocol to avoid the use of high temperature and normally used chemical reductants. Moreover, the asprepared r-GO based conductive cotton fabric showed a reproducible characteristic as a strain sensor. This provides an environmentally-friendly and cost-effective route for scalable fabrication of r-GO based conductive cotton fabrics, paving the way to future natural electronic textiles based graphene and two-dimensional materials.

\section{Acknowledgements}

The authors are grateful for the financial support of the National Natural Science Foundation of China (21174055) and Research Innovation Program for College 
Graduates of Jiangsu Province (SJZZ15_0147). TC and FT acknowledge funding from the European Commission through the Graphene Flagship and the ERC grant Hetero2D. Many thanks also give to Wuxi Graphene Industry Development Demonstration Area Detection Centre for FE-SEM, SPM and Raman analysis and the useful discussion.

\section{References}

[1] A.M. Gaikwad, A.M. Zamarayeva, J. Rousseau, H.W. Chu, I. Derin, D.A. Steingart, Highly Stretchable Alkaline Batteries Based on an Embedded Conductive Fabric, Adv. Mater. 24(37) (2012) 5071-5076, http://dx.doi.org/10.1002/adma.201201329.

[2] L.H. Bao, X.D. Li, Towards Textile Energy Storage from Cotton T-Shirts, Adv. Mater. 24(24) (2012) 3246-3252, http://dx.doi.org/10.1002/adma.201200246.

[3] S. Seyedin, J.M. Razal, P.C. Innis, A. Jeiranikhameneh, S. Beirne, G.G. Wallace, Knitted Strain Sensor Textiles of Highly Conductive All-Polymeric Fibers, ACS Appl. Mater. Interfaces 7(38) (2015) 21150-21158, http://dx.doi.org/10.1021/acsami.5b04892.

[4] A.G. Avila, J.P. Hinestroza, Smart textiles - Tough cotton, Nat. Nanotechnol. 3(8) (2008) 458-459, http://dx.doi.org/10.1038/nnano.2008.233.

[5] M. Pasta, F. La Mantia, L. Hu, H.D. Deshazer, Y. Cui, Aqueous supercapacitors on conductive cotton, Nano Res. 3(6) (2010) 452-458, http://dx.doi.org/10.1007/s12274-010-0006-8.

[6] Y. Li, Y.A. Samad, K. Liao, From cotton to wearable pressure sensor, J. Mater. Chem. A 3(5) (2015) 2181-2187, http://dx.doi.org/10.1039/c4ta05810k.

[7] L. Hu, M. Pasta, F.L. Mantia, L. Cui, S. Jeong, H.D. Deshazer, et al. Stretchable, Porous, and Conductive Energy Textiles, Nano Lett. 10(2) (2010) 708-714, http://dx.doi.org/ 10.1021/n1903949m.

[8] D. Roy, M. Semsarilar, J.T. Guthrie, S. Perrier, Cellulose modification by polymer grafting: a review, Chem. Soc. Rev. 38(7) (2009) 2046-2064, http://dx.doi.org/10.1039/b808639g. 
[9] M.R. Nateghi, M. Shateri-Khalilabad, Silver nanowire-functionalized cotton fabric, Carbohydr. Polym. $117 \quad$ (2015) 160-168, http://dx.doi.org/10.1016/j.carbpol.2014.09.057.

[10] Y. Cheng, R.R. Wang, J. Sun, L. Gao, Highly Conductive and Ultrastretchable Electric Circuits from Covered Yarns and Silver Nanowires, ACS Nano 9(4) (2015) 3887-3895, http://dx.doi.org/10.1021/nn5070937.

[11] K. Firoz Babu, P. Dhandapani, S. Maruthamuthu, M. Anbu Kulandainathan, One pot synthesis of polypyrrole silver nanocomposite on cotton fabrics for multifunctional property, Carbohydr. Polym. 90(4) (2012) 1557-1563, http://dx.doi.org/10.1016/j.carbpol.2012.07.030.

[12] A. Razaq, M.H. Asif, R. Kalsoom, A.F. Khan, M.S. Awan, S. Ishrat, et al. Conductive and electroactive composite paper reinforced by coating of polyaniline on lignocelluloses fibers, J. Appl. Polym. Sci. 132(29) (2015) 42293, http://dx.doi.org/10.1002/app.42293.

[13] Y.F. Li, R.Y. Qian, Stability of Conducting Polymers from the Electrochemical Point-Of-View, Synthetic Met. 53(2) (1993) 149-154, http://dx.doi.org/10.1016/0379-6779(93)90886-2.

[14] F. Torrisi, J.N. Coleman, Electrifying inks with 2D materials, Nat. Nanotechnol. 9(10) (2014) 738-739, http://dx.doi.org/10.1038/nnano.2014.218.

[15] S.V. Morozov, K.S. Novoselov, M.I. Katsnelson, F. Schedin, D.C. Elias, J.A. Jaszczak, et al. Giant intrinsic carrier mobilities in graphene and its bilayer, Phys. Rev. $\quad$ Lett. 100(1) 016602, http://dx.doi.org/10.1103/PhysRevLett.100.016602.

[16] C. Lee, X.D. Wei, J.W. Kysar, J. Hone, Measurement of the elastic properties and intrinsic strength of monolayer graphene, Science 321(5887) (2008) 385-388, http://dx.doi.org/10.1126/science.1157996.

[17] D.R. Dreyer, S. Park, C.W. Bielawski, R.S. Ruoff, The chemistry of graphene oxide, Chem. Soc. Rev. 39(1) (2010) 228-240, http://dx.doi.org/10.1039/b917103g. 
[18] S. Stankovich, R.D. Piner, S.T. Nguyen, R.S. Ruoff, Synthesis and exfoliation of isocyanate-treated graphene oxide nanoplatelets, Carbon 44(15) (2006) 3342-3347, http://dx.doi.org/10.1016/j.carbon.2006.06.004

[19] C. Mattevi, G. Eda, S. Agnoli, S. Miller, K.A. Mkhoyan, O. Celik, et al. Evolution of Electrical, Chemical, and Structural Properties of Transparent and Conducting Chemically Derived Graphene Thin Films, Adv. Funct. Mater. 19(16) (2009) 2577-2583, http://dx.doi.org/10.1002/adfm.200900166.

[20] L.L. Xu, M.X. Guo, S. Liu, S.W. Bian, Graphene/cotton composite fabrics as flexible electrode materials for electrochemical capacitors, RSC Adv. 5(32) (2015) 25244-25249, http://dx.doi.org/10.1039/C4RA16063K.

[21] W.W. Liu, X.B. Yan, J.W. Lang, C. Peng, Q.J. Xue, Flexible and conductive nanocomposite electrode based on graphene sheets and cotton cloth for supercapacitor, J. Mater. Chem. 22(33) (2012) 17245, http://dx.doi.org/10.1039/C2JM32659K.

[22] I.A. Sahito, K.C. Sun, A.A. Arbab, M.B. Qadir, S.H. Jeong, Graphene coated cotton fabric as textile structured counter electrode for DSSC, Electrochim. Acta 173 (2015) 164-171, http://dx.doi.org/10.1016/j.electacta.2015.05.035.

[23] K. Javed, C.M.A. Galib, F. Yang, C.-M. Chen, C. Wang, A new approach to fabricate graphene electro-conductive networks on natural fibers by ultraviolet curing method, Synthetic Met. $193 \quad$ (2014) 41-47, http://dx.doi.org/10.1016/j.synthmet.2014.03.028.

[24] C. Zhao, K. Shu, C. Wang, S. Gambhir, G.G. Wallace, Reduced graphene oxide and polypyrrole/reduced graphene oxide composite coated stretchable fabric electrodes for supercapacitor application, Electrochim. Acta 172 (2015) 12-19, http://dx.doi.org/10.1016/j.electacta.2015.05.019.

[25] Y.J. Yun, W.G. Hong, W.-J. Kim, Y. Jun, B.H. Kim, A Novel Method for Applying Reduced Graphene Oxide Directly to Electronic Textiles from Yarns to Fabrics, Adv. Mater. 25(40) (2013) 5701-5705, http://dx.doi.org/10.1002/adma.201303225. 
[26] L. Karimi, M.E. Yazdanshenas, R. Khajavi, A. Rashidi, M. Mirjalili, Using graphene/TiO2 nanocomposite as a new route for preparation of electroconductive, self-cleaning, antibacterial and antifungal cotton fabric without toxicity, Cellulose 21(5) (2014) 3813-3827, http://dx.doi.org/10.1007/s10570-014-0385-1.

[27] M. Shateri-Khalilabad, M.E. Yazdanshenas, Fabricating electroconductive cotton textiles using graphene, Carbohydr. Polym. 96(1) (2013) 190-195, http://dx.doi.org/10.1016/j.carbpol.2013.03.052.

[28] Y.W. Zhu, M.D. Stoller, W.W. Cai, A. Velamakanni, R.D. Piner, D. Chen, et al. Exfoliation of Graphite Oxide in Propylene Carbonate and Thermal Reduction of the Resulting Graphene Oxide Platelets, ACS Nano 4(2) (2010) 1227-1233, http://dx.doi.org/10.1021/nn901689k.

[29] Z. Xu, Y. Liu, X. Zhao, L. Peng, H. Sun, Y. Xu, et al. Ultrastiff and Strong Graphene Fibers via Full-Scale Synergetic Defect Engineering, Adv. Mater. (2016) n/a-n/a. http://dx.doi.org/10.1002/adma.201506426

[30] B. Zhao, P. Liu, Y. Jiang, D.Y. Pan, H.H. Tao, J.S. Song, et al. Supercapacitor performances of thermally reduced graphene oxide, J. Power Sources 198 (2012) 423-427, http://dx.doi.org/10.1016/j.jpowsour.2011.09.074.

[31] D.C. Marcano, D.V. Kosynkin, J.M. Berlin, A. Sinitskii, Z.Z. Sun, A. Slesarev, et al. Improved Synthesis of Graphene Oxide, ACS Nano 4(8) (2010) 4806-4814, http://dx.doi.org/10.1021/nn1006368.

[32] Z. Bo, X.R. Shuai, S. Mao, H.C. Yang, J.J. Qian, J.H. Chen, et al. Green preparation of reduced graphene oxide for sensing and energy storage applications, Sci. Rep-Uk. 4 (2014), http://dx.doi.org/10.1038/srep04684.

[33] M.W. Tian, X.L. Hu, L.J. Qu, S.F. Zhu, Y.N. Sun, G.T. Han, Versatile and ductile cotton fabric achieved via layer-by-layer self-assembly by consecutive adsorption of graphene doped PEDOT: PSS and chitosan, Carbon 96 (2016) 1166-1174, http://dx.doi.org/10.1016/j.carbon.2015.10.080.

[34] S. Stankovich, D.A. Dikin, R.D. Piner, K.A. Kohlhaas, A. Kleinhammes, Y. Jia, et al. Synthesis of graphene-based nanosheets via chemical reduction of exfoliated 
graphite $\quad$ oxide, $\quad$ Carbon 45(7) (2007) 1558-1565, http://dx.doi.org/10.1016/j.carbon.2007.02.034.

[35] W. Chen, L. Yan, P.R. Bangal, Preparation of graphene by the rapid and mild thermal reduction of graphene oxide induced by microwaves, Carbon 48(4) (2010) 1146-1152, http://dx.doi.org/10.1016/j.carbon.2009.11.037.

[36] H. Varela-Rizo, I. Rodriguez-Pastor, C. Merino, I. Martin-Gullon, Highly crystalline graphene oxide nano-platelets produced from helical-ribbon carbon nanofibers, Carbon 48(12) (2010) 3640-3643, http://dx.doi.org/10.1016/j.carbon.2010.05.033.

[37] A.C. Ferrari, J.C. Meyer, V. Scardaci, C. Casiraghi, M. Lazzeri, F. Mauri, et al. Raman Spectrum of Graphene and Graphene Layers, Phys. Rev. Lett. 97(18) (2006) 187401, http://dx.doi.org/10.1103/PhysRevLett.97.187401.

[38] F. Tuinstra, J. L. Koenig, Raman spectrum of graphite, J. Chem. Phys. 53(3) (1970) 1126-1130, http://dx.doi.org/10.1063/1.1674108.

[39] A.C. Ferrari, J. Robertson, Resonant Raman spectroscopy of disordered, amorphous, and diamond like carbon, Phys. Rev. B 64(7) (2001) 075414, http://dx.doi.org/10.1103/PhysRevB.64.075414.

[40] E.H.M. Ferreira, M.V.O. Moutinho, F. Stavale, M.M. Lucchese, R.B. Capaz, C.A. Achete, et al. Evolution of the Raman spectra from single-, few-, and many-layer graphene with increasing disorder, Phys. Rev. B 82(12) (2010) 125429, http://dx.doi.org/10.1103/PhysRevB.82.125429.

[41] K.N. Kudin, B. Ozbas, H.C. Schniepp, R.K. Prud'homme, I.A. Aksay, R. Car, Raman spectra of graphite oxide and functionalized graphene sheets, Nano Lett. 8(1) (2008) 36-41, http://dx.doi.org/10.1021/n1071822y.

[42] A.C. Ferrari, J. Robertson, Interpretation of Raman spectra of disordered and amorphous carbon, Phys. Rev. B 61(20) (2000) 14095-14107, http://dx.doi.org/10.1103/PhysRevB.61.14095.

[43] K.H. Wu, H.H. Cheng, A.A. Mohammad, I. Blakey, K. Jack, I.R. Gentle, et al. Electron-beam writing of deoxygenated micro-patterns on graphene oxide film, Carbon 95 (2015) 738-745, http://dx.doi.org/10.1016/j.carbon.2015.08.116. 
[44] S. Pei, H.M. Cheng, The reduction of graphene oxide, Carbon 50(9) (2012) 32103228, http://dx.doi.org/10.1016/j.carbon.2011.11.010.

[45] H.J. Shin, K.K. Kim, A. Benayad, S.-M. Yoon, H.K. Park, I.-S. Jung, et al. Efficient Reduction of Graphite Oxide by Sodium Borohydride and Its Effect on Electrical Conductance, Adv. Funct. Mater. 19(12) (2009) 1987-1992, http://dx.doi.org/10.1002/adfm.200900167.

[46] S. Pei, J. Zhao, J. Du, W. Ren, H.M. Cheng, Direct reduction of graphene oxide films into highly conductive and flexible graphene films by hydrohalic acids, Carbon 48(15) (2010) 4466-4474, http://dx.doi.org/10.1016/j.carbon.2010.08.006.

[47] X.F. Gao, J. Jang, S. Nagase, Hydrazine and Thermal Reduction of Graphene Oxide: Reaction Mechanisms, Product Structures, and Reaction Design, J. Phys. Chem. C 114(2) (2010) 832-842, http://dx.doi.org/10.1021/jp909284g.

[48] C. Valles, J.D. Nunez, A.M. Benito, W.K. Maser, Flexible conductive graphene paper obtained by direct and gentle annealing of graphene oxide paper, Carbon 50(3) (2012) 835-844, http://dx.doi.org/10.1016/j.carbon.2011.09.042.

[49] X. Li, R.J. Zhang, W.J. Yu, K.L. Wang, J.Q. Wei, D.H. Wu, A.Y. Cao, Z.H. Li, Y. Cheng, Q.S. Zheng, R.S. Ruoff, H.W. Zhu, Stretchable and highly sensitive graphene-on-polymer strain sensors, Sci. Rep-Uk. 2 (2012), http://dx.doi.org/10.1038/srep00870.

[50] T. Vervust, G. Buyle, F. Bossuyt, J. Vanfleteren, Integration of stretchable and washable electronic modules for smart textile applications, J. Text. I. 103(10) (2012) 1127-1138, http://dx.doi.org/10.1080/00405000.2012.664866.

[51] D. Van Lam, K. Jo, C.H. Kim, S. Won, Y. Hwangbo, J.H. Kim, et al. Calligraphic ink enabling washable conductive textile electrodes for supercapacitors, J. Mater. Chem. A 4(11) (2016) 4082-4088, http://dx.doi.org/10.1039/c6ta01341d.

[52] N. Nasirizadeh, M. Dehghani, M.E. Yazdanshenas, Preparation of hydrophobic and conductive cotton fabrics using multi-wall carbon nanotubes by the sol-gel method, J. Sol-Gel Sci. Techn. 73(1) (2015) 14-21, http://dx.doi.org/10.1007/s10971-014-3488-0. 